\title{
Graph Analysis and Visualization for Brain Function Characterization Using EEG Data
}

\author{
Vangelis Sakkalis ${ }^{\mathbf{a}, *}$, Vasilis Tsiaras ${ }^{\mathbf{a}, \mathbf{b}}$, and Ioannis G. Tollis ${ }^{\mathbf{a}, \mathbf{b}}$ \\ ${ }^{a}$ Biomedical Informatics Lab, Institute of Computer Science, Foundation for Research \\ and Technology, Heraklion, Greece \\ ${ }^{b}$ Department of Computer Science, University of Crete, Heraklion, Greece
}

\begin{abstract}
Over the past few years, there has been an increased interest in studying the underlying neural mechanism of cognitive brain activity as well as in diagnosing certain pathologies. Noninvasive imaging modalities such as functional magnetic resonance imaging (fMRI), positron emission tomography (PET), and dynamic signal acquisition techniques such as quantitative electroencephalography (EEG) have been vastly used to estimate cortical connectivity and identify functional interdependencies among synchronized brain lobes. In this area, graph-theoretic concepts and tools are used to describe large scale brain networks while performing cognitive tasks or to characterize certain neuropathologies. Such tools can be of particular value in basic neuroscience and can be potential candidates for future inclusion in a clinical setting. This paper discusses the application of the high time resolution EEG to resolve interdependence patterns using both linear and nonlinear techniques. The network formed by the statistical dependencies between the activations of distinct and often well separated neuronal populations is further analyzed using a number of graph theoretic measures capable of capturing and quantifying its structure and summarizing the information that it contains. Finally, graph visualization reveals the hidden structure of the networks and amplifies human understanding. A number of possible applications of the graph theoretic approach are also listed. A freely available standalone brain visualization tool to benefit the healthcare engineering community is also provided (http://www.ics.forth.gr/bmi/tools.html).
\end{abstract}

Keywords: EEG; synchronization; brain dynamics; brain functional networks; graph measures; random graph models

\section{INTRODUCTION}

The brain is a complex dynamical system in which information is continuously processed and transferred to other interconnected regions to make up a functional network [1-5]. Functional networks are thought to provide the physiological basis for

*Corresponding author. Address: BMI lab, Institute of Computer Science (ICS), Foundation for Research and Technology (FORTH), Heraklion 71110, Greece. E-mail address: sakkalis@ics.forth.gr (V. Sakkalis). 
information processing and mental representations [6,7], and have been studied across different conditions of rest $[8,9,10]$ or cognitive load [11].

Studies with detailed electroencephalography (EEG) and magnetoencephalography (MEG) signals have revealed local synchronization patterns and cortico-cortical interactions involved in several cognitive functions [12], with composite subtasks being triggered within different brain regions by unitary brain sources that subsequently synchronize to complete the task. Thus, the dynamics of interaction among different EEG/ MEG channels may be used for indexing neural synchrony of such local or distant brain sources [13]. Such sources act synchronously behaving similar to coupled oscillators [14] and their interactions can be measured using pair-wise linear (cross-coherence or phasecoherence) [16] or nonlinear dynamics and models [15, 16, 17]. Furthermore, the causality of the functional coupling of such oscillatory activities can be assessed with partially directed coherence, which reveals the direction of statistically significant relationships [18]. Synchronization can be evaluated not only on the actual recordings on the scalp electrodes but also on independent components. The later are derived from linear un-mixing transforms and are free from volume conduction effects [15, 16].

Networks are modeled by graphs which consist of a set of vertices and a set of pair of vertices called edges (Figure 1). In this respect, graph theory offers a unique perspective and a common framework for studying interactions between local and remote cortical areas, where areas correspond to vertices and interactions to edges. These interactions can be estimated from multivariate EEG time series of brain activity where each vertex is identified with a scalar time series, and there is an edge between two vertices if and only if statistical relationship is inferred between the corresponding time series, as described above. Whether an edge exists between the corresponding vertices is a very informative representation of the entire system, although each interaction depends on time, space, cognitive task and many other intrinsic details. Especially during the last decade, it became evident that brain functional networks as well as brain anatomical networks are characterized by the same topological properties that are present in most real networks, as for instance relatively small characteristic path lengths (average length over all shortest paths between each pair of vertices), high clustering coefficients, fat tailed shapes in the degree distributions, degree correlations, and the presence of motifs and community structures [2, 19].

Brain anatomical and functional networks are neither totally regular nor entirely random. Uncovering the hidden regularities and organizational principles of brain networks often requires comparison with a null model network that has similar statistical properties. A well-fitting network model that reproduces the network structure and/or the laws through which the network has emerged can enable us to understand the underlying processes and to predict the structure and behavior of the brain. Thus far, four null model networks have been considered: the Erdös-Rényi random graph, the small world, the scale free, and the geometric random graph models.

The information contained in a network can be summarized with graph measures. Graph measures have been applied to topological analysis of brain functional networks, and many of them have been shown to reflect disease and statistically significant differences between healthy subjects and subjects with neuropathologies such as 


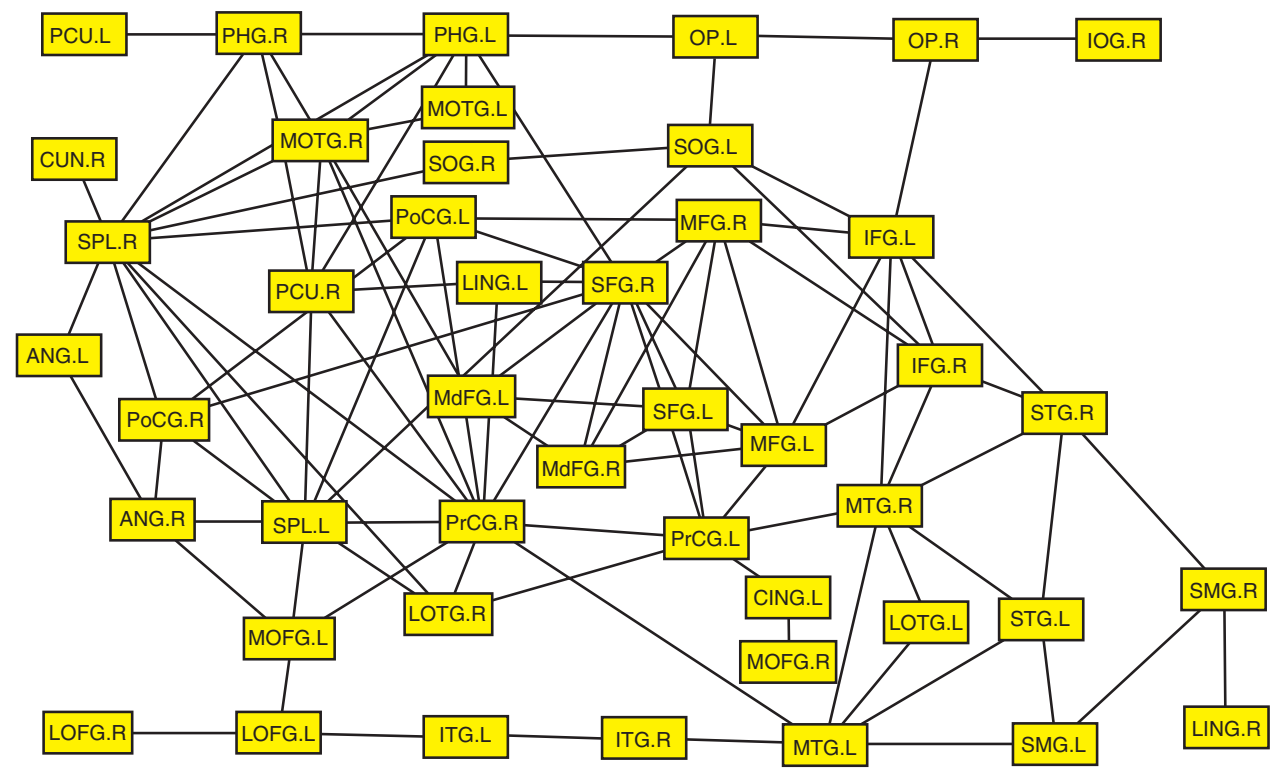

Figure 1. Large-scale anatomical network in the human brain that consists of 45 areas [46]. Two areas are considered anatomically connected if they show statistically significant correlations in cortical thickness measurements from magnetic resonance images. This network is modeled as a graph which consists of vertices (yellow rectangles) and edges (line segments between pairs of vertices).

epilepsy, Alzheimer's disease, autism, Parkinson's disease and schizophrenia [20, 21, $22,23,24]$. All these diseases have been associated with abnormal neural synchronization, and as a result, with functional networks that systematically differ from those of control subjects. Epilepsy has been associated with too high and too extended neural synchronization [25, 26]. Patients with Alzheimer's disease show reduced synchronization in the alpha and beta frequency bands [27, 28, 29]. Cognitive dysfunctions associated with autism are explained with reduced functional connectivity and neural synchronization $[30,31,32]$. There are an increasing amount of data linking impaired motor processing in Parkinson's disease with excessive synchrony at low beta frequencies in basal ganglia-cortical loops [33]. Concerning schizophrenia, there is a growing body of evidence that the clinical symptoms and cognition dysfunctions observed in schizophrenia are caused by a disturbance in connectivity between different brain regions. In particular, there is reduction in both local and long-range synchronization [34, 35, 36, 37]. Additionally, a number of studies demonstrate that there is a strong negative association between the characteristic path length (the average shortest path lengths between each pair of vertices) of the resting-state brain functional network and the intelligence quotient (IQ), suggesting that human intellectual 
performance is likely to be related to how efficiently our brain integrates information between multiple brain regions [38].

The purpose of this paper is to review the most robust linear and nonlinear synchronization measures for constructing functional networks including measures for comparing and charactering networks. It also stresses the significance of network measures as potential biomarkers of brain pathophysiology.

This paper proceeds as follows: Section 2.1 provides an introductory review of basic graph concepts and indices that are further utilized in describing the functional connectivity of a brain network, discussed in sections 2.2 and 2.3. Section 2.4 covers network models, and section 2.5 briefly reviews the most robust and popular linear and nonlinear methods for quantifying synchronization between time series pointing to references for further detailed information. Section 3 presents real case application paradigms, while section 4 concludes this paper.

\section{METHODS}

\subsection{Preliminaries}

A recent trend in brain functional connectivity analysis is to model the interdependencies among brain signals with networks [7, 39]. Interdependence among different brain areas is estimated from multivariate neurophysiological signals (EEG, MEG, electrocorticography $(\mathrm{ECoG})$ ) and/or haemodynamic response images (functional magnetic resonance imaging (fMRI)). Then a network is formed by corresponding brain areas or channels to vertices and by considering an edge between two vertices if and only if the estimated interdependence is above a threshold (Figure 1).

The next step in the analysis is to measure some statistics to characterize the network. Using the network characterization, one can draw conclusions on the effect of illnesses or of cognitive loads on functional connectivity [9, 28, 41, 42]. Figure 2 outlines a basic signal preprocessing and analysis schema.

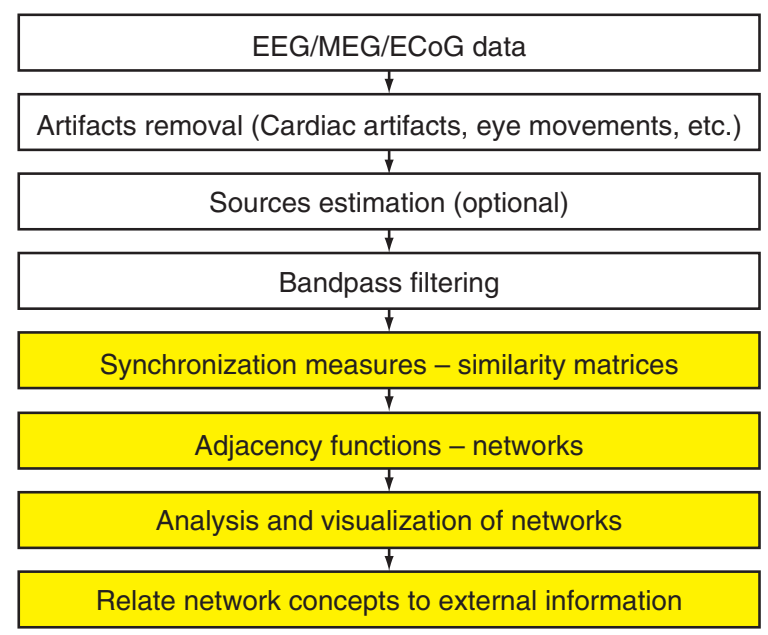

Figure 2. Sample outline of neurophysiological signals analysis. 
A graph $G=(V, E)$ is a pair of vertices $V=\left\{v_{1}, v_{2}, \ldots, v_{n}\right\}$ and edges $E=\left\{e_{1}, e_{2}, \ldots\right.$, $\left.e_{m}\right\}$, where each edge $e \in E$ is an ordered or unordered pair of vertices. An ordered pair $e=(u, v) \in V \times V$ is called a directed edge, while an unordered pair $e=\{u, v\}$, where $u, v \in V$, is called an undirected edge. In case $u=v, e$ is called a directed or undirected loop. In our study, we consider simple graphs without self-loops and multiple edges. Also, the cardinality of $V$ is denoted by $n$ (i.e., $n=|V|$ ). The two vertices joined by an edge are called its endvertices. In the undirected case, if two vertices $u, v$ are joined by an edge $e=\{u, v\}$, they are adjacent and called neighbors. The degree of a vertex $v \in V$, denoted by $\operatorname{deg}(v)$, is the number of edges in $E$ that have $v$ as an endvertex. The sets of neighbors of vertex $v \in V$ is denoted by $\mathrm{N}(v)=\{u \in V \mid\{u, v\} \in E\}$. In the directed case, if $e=(u, v) \in E$ is a directed edge, we say that vertex $u$ is the origin of $e$ and vertex $v$ is the destination of $e$. The origin of $e$ is denoted by $\operatorname{orig}(e)$ and the destination of $e$ by $\operatorname{dest}(e)$. The out-degree of $v \in V$, denoted by $\operatorname{deg}^{+}(v)$, is the number of edges in $E$ that have origin $v$. The in-degree of $v \in V$, denoted by $\operatorname{deg}^{-}(v)$, is the number of edges with destination $v$. The degree of $v \in V$, denoted by $\operatorname{deg}(v)$, is the number of edges with origin or destination $v\left(\operatorname{deg}(v)=\operatorname{deg}^{+}(v)+\operatorname{deg}^{-}(v)\right)$. For every vertex $v \in V, N^{+}(v)=\{u$ $\in V \mid(v, u) \in E\}$ and $N^{-}(v)=\{u \in V \mid(u, v) \in E\}$ are the sets of out-neighbors and inneighbors of vertex $v$, respectively.

A path from $v_{0}$ to $v_{k}$ in a graph $G=(V, E)$ is an alternating sequence $v_{0}, e_{1}, v_{1}, e_{2}$, $v_{2}, \ldots, e_{k}, v_{k}$ of vertices and edges such that $e_{i} \neq e_{j}$ for $i \neq j$, where $e_{i}=\left\{v_{i-1}, v_{i}\right\}$ in the undirected case and $e_{i}=\left\{v_{i-1}, v_{i}\right\}$ in the directed case. The length of a path is defined as the number of edges on the path. There is always a zero length path from $v_{0}$ to $v_{0}$. The shortest path distance from vertex $u$ to vertex $v$ is denoted by $d_{G}(u, v)$. If vertex $v$ is unreachable from vertex $u$, then $d_{G}(u, v)=+\infty$.

\subsection{Graph Metrics}

Networks are often classified into unifying categories in order to obtain a better understanding of their structure and function. In order to classify a network, it is necessary to reduce the information by describing essential properties of the network with a few numbers (often called network metrics, measures, or statistics). Network metrics are chosen to catch the relevant and needed information and to differentiate between certain classes of networks. They are easily computable in order to be useful in several algorithms and applications.

\section{Average Degree}

The mean degree of a graph is the average degree over all vertices, thus

$$
K=\frac{1}{n} \sum_{v \in V} \operatorname{deg}(v)
$$

The average degree of graph of Figure 1 is 4.53 .

\section{Clustering Coefficient}

The clustering coefficient $C(v)$ for a vertex $v$ is the proportion of links between the vertices within its neighborhood divided by the number of links that could possibly 
exist between them. For an undirected graph, if a vertex $v_{i}$ has degree $k_{i}, \frac{k_{i}\left(k_{i}-1\right)}{2}$ edges could exist among the vertices within its neighborhood, thus

$$
C\left(v_{i}\right)=\frac{2}{k_{i}\left(k_{i}-1\right)}\left|\left\{e=\left\{v_{j}, v_{k}\right\} \mid v_{j}, v_{k} \in N\left(v_{i}\right)\right\}\right|
$$

This measure is 1 if every neighbor connected to $v_{\mathrm{i}}$ is also connected to every other vertex within the neighborhood, and 0 if no vertex that is connected to $v_{i}$ connects to any other vertex that is connected to $v_{i}$. The clustering coefficient for a graph is given by Watts and Strogatz [43] as the average of the clustering coefficient for each vertex,

$$
C=\frac{1}{n} \sum_{v \in V} C(v)
$$

and is a measure of the tendency of graph vertices to form local clusters. For example, in the graph of Figure 1, in order to compute the cluster coefficient for vertex STG.L, we first determine the other vertices to which it is directly connected. These neighbors are vertices STG.R, MTG.R, MTG.L and SMG.L. Then we determine how many edges exist in the set of neighbors. In this case, the edges among neighbors of vertex STG.L are 3 edges: $\{S T G . R, M T G . R\},\{M T G . L, M T G . R\},\{M T G . L, S M G . L\}$. Next, we determine how many edges could have existed between the neighbors. In this case, there could be 6 edges (one edge for every pair of vertices STG.R, MTG.R, MTG.L, $S M G . Y$ ). The clustering coefficient of vertex STG.L is the ratio of $3 / 6=0.5$. The clustering coefficient of the graph of Figure 1 is 0.299 .

\section{Eccentricity, Radius and Diameter of a Graph}

There are several measures defined in terms of distance, such as the eccentricity of a vertex, the radius, and the diameter of a graph. The eccentricity $\varepsilon(v)$ of a vertex $v$ in a connected graph $G$ is the maximum graph distance between $v$ and any other vertex $u$ of $G[44,45]$

$$
\varepsilon(v)=\left|\max \left\{d_{G}(u, v) \mid u \in V\right\}\right|
$$

For a disconnected graph, all vertices are defined to have infinite eccentricity. The radius and the diameter of a graph are the minimum and the maximum eccentricity of any vertex in the graph, respectively. The diameter represents the greatest distance between any two vertices.

Average Shortest Path Length

The average shortest path length

$$
L=\frac{1}{n(n-1)} \sum_{u, v \in V, u \neq \nu} d_{G}(u, v)
$$


is the average shortest path (distance) connecting any two vertices of the graph and is a measure of the interconnectedness of the graph. Note that the absence of a path between $u$ and $v$ implies $d_{G}(u, v)=+\infty$. In practice, we set $d_{G}(u, v)=M \gg>n$, when $d_{G}(u, v)=+\infty$.

\section{Network Efficiency}

One drawback of the average shortest path length is that it can only be used for connected graphs. A measure closely related to the average shortest path length is the network efficiency:

$$
E_{f}=\frac{1}{n(n-1)} \sum_{u, v \in V, u \neq v} \frac{1}{d_{G}(u, v)}
$$

Network efficiency is the inverse of the harmonic mean whereas the average shortest path length is the arithmetic mean of the shortest path distances. Note that eqn. (6) can also be used for disconnected graphs. If some vertices $v$ and $u$ are not connected, they do not contribute to $E_{f}\left(d_{G}(v, u)=+\infty \Rightarrow \frac{1}{d_{G}(v, u)}=0\right)$.

Network efficiency quantifies the effectiveness of information flow within brain networks. He et al. showed that in multiple sclerosis, the efficiency of the anatomical network is reduced in a manner proportional to the extent of total white matter lesions [46]. Achard and Bullmore found that the resting state functional networks provide high efficiency of information processing for low anatomical connection [47]. Efficiency was reduced in older people and the detrimental effects of age to efficiency were localized to frontal and temporal cortical and subcortical regions [47]. The efficiency of graph in Figure 1 is 0.407.

\section{Assortativity}

An important network feature is the similarity between properties of adjacent network vertices. Newman [48, 49] proposed a measure to quantify the degree of similarity (dissimilarity) between adjacent vertices in a network using assortative mixing, which is given by correlation between the degrees of every pair of adjacent vertices. The assortativity coefficient for an undirected graph is defined as the (sample) Pearson product-moment correlation coefficient written in a symmetrical form:

$$
r=\frac{4|E| \sum_{\{u, v\} \in E} \operatorname{deg}(u) \operatorname{deg}(v)-\left(\sum_{\{u, v\} \in E}(\operatorname{deg}(u)+\operatorname{deg}(v))\right)^{2}}{2|E| \sum_{\{u, v\} \in E}\left(\operatorname{deg}(u)^{2}+\operatorname{deg}(v)^{2}\right)-\left(\sum_{\{u, v\} \in E}(\operatorname{deg}(u)+\operatorname{deg}(v))\right)^{2}}
$$

where $|E|$ denotes the number of edges. The assortativity coefficient $r$ lies between -1 and 1 , whereby $r=1$ means perfect assortativeness, $r=-1$ means perfect disassortativeness, and $r=0$ means no assortativeness (random linking). A special case of assortative mixing according to a scalar vertex property is mixing according to vertex degree. 
Many social networks are assortative since vertices having many connections tend to connect with other highly connected vertices [50]. On the other hand, most technological and biological networks are disassortative since they have the property that vertices with high degree are preferably connected with ones of low degree [51]. Exceptions are the protein contact networks and the brain functional networks which are assortative $[52,53]$. Disassortative mixing observed in certain biological networks (metabolic signaling pathways network, and gene regulatory network) is conjectured to be responsible for decreasing the likelihood of crosstalk between different functional modules of the cell, and increasing the overall robustness of a network by localizing effects of deleterious perturbations [54]. On the other hand, from computational studies, it has been observed that information can be easily transferred through assortative networks as compared to that in disassortative networks $[48,49,55]$. Note that the network of Figure 1 is assortative having an assortativity coefficient of 0.083 .

\subsection{Centrality Measures}

Characterizing local properties of networks is important both in theory and practice since at the local scale, we can detect which vertices are the most relevant to the organization and functioning of a network. These local measures are commonly named centrality measures (or centrality indices), and have proven of great value in analyzing the role played by individuals in social networks and in identifying essential proteins [56], keystone species [57], and functionally important brain regions [58].

There is no commonly accepted definition for centrality. Many authors introduced their own centrality, avoiding a strict definition for centrality in general. The intuition about a centrality measure is that it denotes an order of importance on the vertices or edges of a graph by assigning real values to them. As a minimal requirement, most centrality measures depend only on the structure of a graph.

\section{Degree Centrality}

The simplest and most widely studied centrality is the degree centrality $C_{D}(v)$ of a vertex $v$ which for an undirected graph is simply defined as the degree $\operatorname{deg}(v)$ of $v$. Degree centrality is normalized to range $[0,1]$ by dividing $\operatorname{deg}(v)$ with the maximum possible degree $(n-1)$.

$$
C_{D}(v)=\frac{\operatorname{deg}(v)}{n-1}
$$

In directed networks, two variants of the degree centrality may be appropriate: the in-degree centrality $C_{\mathrm{D}}^{-}(v)=\operatorname{deg}^{-}(v) /(n-1)$ and the out-degree centrality $C_{D}^{+}(v)=$ $\operatorname{deg}^{+}(v) /(n-1)$.

Degree is the most fundamental network measure and most other measures are linked to vertex degree. The degree sequence is argued to reflect some fundamental aspects of natural, social and technological networks [59]. Manke et al. [60] advocate the view that the degree can be considered as correlation of underlying dynamical properties, such as the stability of a dynamic process to random perturbations. In the 
graph of Figure 1 we have $\operatorname{deg}(\operatorname{PrCG} \cdot R)=11, \operatorname{deg}(S P L \cdot R)=11$ and $\operatorname{deg}(S F G \cdot R)=11$. The corresponding normalized centralities are $C_{D}(\operatorname{Pr} C G . R)=0.25, C_{D}(S P L . R)=0.25$ and $C_{D}(S F G . R)=0.25$.

\section{Shortest-Path Efficiency}

Latora and Marchiori $[61,62]$ defined the efficiency $e f_{v u}$ in the communication between vertices $v$ and $u$ to be inversely proportional to the shortest distance, $1 / d_{G}(v, u)$. Then the average efficiency of vertex $v$ is given by:

$$
C_{E f}(v)=\frac{1}{n-1} \sum_{u \in V \backslash\{v\}} \frac{1}{d_{G}(v, u)}
$$

Note that eqn. (9) can also be used for disconnected graphs. If some vertices $v$ and $u$ are not connected, they do not contribute to $C_{E f}(v)\left(d_{G}(v, u)=+\infty \Rightarrow \frac{1}{d_{G}(v, u)}=0\right)$.

Shortest-Path Betweenness Centrality

The shortest-path betweenness centrality $C_{B}(v)$ of a vertex $v \in V$ is defined to be $[63,64]$

$$
C_{B}(v)=\frac{1}{(n-1)(n-2)} \sum_{s \in V \backslash\{v\}} \sum_{t \in V \backslash\{v, s\}} \frac{\sigma_{s t}(v)}{\sigma_{s t}}
$$

where $\sigma_{s t}$ is the number of shortest $(s, t)$-paths, and let $\sigma_{s t}(v)$ be the number of shortest $(s, t)$-paths passing through some vertex $v$ other than $s, t$. The relative numbers $\frac{\sigma_{s t}(v)}{\sigma_{s t}}$ are interpreted as the extent to which vertex $v$ controls the communication between vertices $s$ and $t$. A vertex is central, if it is between many pairs of other vertices. The definition of betweenness applies to disconnected graphs without modification.

\subsection{Network Models}

The basic idea behind using graphs as the first step in the study of a brain network is that measuring some basic properties of a complex network can help us in understanding its structure. Brain anatomical and functional networks as well as biological, social and technological networks display non-trivial topological features, with patterns of connection between their elements that are neither totally regular nor totally random. Uncovering the hidden regularities and organizational principles of brain networks often requires comparison with a null model network that has similar statistical properties. Well established network models which have been extensively used as null models are the Erdös-Rényi random graph, the small world, the scale free, and the geometric random graph models. These are briefly mentioned below:

- Erdös-Rényi (random graph) model: Erdös and Rényi introduced the random graph model and initiated a large area of research $[65,66,67,68]$. There are two closely related variants of the Erdös-Rényi random graph model. In the G(n, m) 
model, a graph is chosen uniformly at random from the collection of all graphs which have $n$ vertices and $m$ edges. In the $G(n, q)$ model, a graph is thought to be constructed by connecting vertices randomly. Each edge is included in the graph with probability $q$, with the presence or absence of any two distinct edges in the graph being independent. The random graph model fails to adequately represent brain networks since they have, among other properties, low clustering coefficient whereas both anatomical and functional networks have high clustering coefficient $[7,47,69]$.

- $\quad$ Scale-free model: The scale-free network model is characterized by its degree distribution, viz. the probability distribution of the degrees over the whole network, following a power law. The power law implies that the degree distribution of these networks has no characteristic scale and that there is a small number of very highly connected vertices called hubs. Recent interest in scalefree networks started in 1999 by Barabási, et al. [70, 71] who proposed a mechanism to explain the appearance of the power-law distribution in a stochastic growth model in which new vertices are added continuously and they preferentially attach to existing vertices with probability proportional to the degree of the target vertex. Many real world networks have power-law degree distributions, such as the World Wide Web [72], the metabolic reaction networks [73] and the protein networks [74]. There is no consensus in scientific community on whether the degree distribution of brain function or anatomical networks follows either a power law $[69,75]$ or a power law with exponential cut-off $[76,77]$.

- Small-world model: Many real world networks exhibit what is called the smallworld property, viz. the condition that most vertices can be reached from every other vertex through short paths. Erdös-Rényi and scale-free networks also have the small-world property. The network model published by Watts and Strogatz [43] combines the small-world property, like random graphs, with high clustering coefficient like lattices. This network model is also called small-world. In brain network literature, the term small-world refers to this model (viz. networks with small diameter and high clustering coefficient). Both brain functional and anatomical networks are small-world [47, 69].

- Geometric random model: A geometric graph $G(V, \rho)$ with radius $\rho$ is a graph where points in a metric space correspond to vertices, and two vertices are adjacent if the distance between them is at most $\rho$. More details about geometric random graphs can be found in [78]. When the position of vertices in a (possibly high dimensional) Euclidean space is important, the random geometric graph is a good candidate null model. Computational experiments have revealed close matches between key topological properties of Protein-Protein Interaction networks and geometric random graph models [79]. Oikonomou showed that brain functional networks estimated at sensor space from schizophrenia and epilepsy data also share key topological properties with geometric random graphs [80].

Recent articles illustrate that there may be evidence for small world networks in characterizing certain brain pathologies like the Alzheimer disease [28].

\subsection{Interdependence Measures}

Before analyzing graph metrics, the graph itself has to be estimated from data such as EEG. In brain functional networks, each vertex is identified with a brain area and each edge corresponds to statistical dependence in the activities of two brain areas. We 
assume here that the activity of a brain area is measured in a scalar time series. Numerous synchronization techniques have allowed the measurement of both the linear and nonlinear dynamic coupling between different brain regions. In this section, we preview linear and nonlinear methods capable of assessing synchronization between different neural assemblies. Such methods applied in different frequency bands have been successfully used in the past as indices of cerebral engagement in cognitive tasks or brain pathologies [17].

\section{Magnitude squared coherence (MSC)}

MSC (or simply coherence) has been a well-established and traditionally used tool to investigate the linear relation between two signals or EEG channels. Let us suppose that we have two simultaneously measured discrete time series $\mathrm{x}_{i}$ and $y_{i}, i=1 \ldots N$. MSC is the cross spectral density function $S_{x y}(f)$, which is simply derived via the FFT of the cross-correlation, normalized by their individual autospectral density functions. Hence, MSC is calculated using the Welch's method as:

$$
\gamma_{x y}(f)=\frac{\left|\left\langle S_{x y}(f)\right\rangle\right|^{2}}{\left|\left\langle S_{x x}(f)\right\rangle\right|\left|\left\langle S_{y y}(f)\right\rangle\right|}
$$

where $\langle\cdot\rangle$ indicates window averaging. The estimated MSC for a given frequency $f$ ranges between 0 (no coupling) and 1 (maximum linear interdependence).

\section{Partial Directed Coherence (PDC)}

The main advantage of this linear method is that it is able to derive additional information on the "driver and response" relationship between observations. The concept of Granger-causality [81] is based on the commonsense idea that causes precede their effects in time and is formulated in terms of predictability. In a linear framework, Granger-causality is commonly evaluated by fitting Vector Autoregressive Models. Suppose that a set of $n$ simultaneously observed time series $\mathbf{x}(t)=\left[x_{1}(t), \ldots, x_{N}(t)\right]^{T}$ is adequately represented by a Vector Autoregressive Model of order $p(\operatorname{MVAR}(\mathrm{p}))$ :

$$
\mathbf{x}(t)=\sum_{k=1}^{p} A_{k} \mathbf{x}(t-k)+\mathbf{w}(t)
$$

where $\mathbf{A}_{k}=\left\lfloor\begin{array}{ccc}a_{11}(k) & \cdots & a_{1 n}(k) \\ \vdots & \ddots & \vdots \\ a_{n 1}(k) & \cdots & a_{n n}(k)\end{array}\right\rfloor$ is the coefficient matrix at time lag $k$, and

$\mathbf{w}(t)=\left[w_{1}(t), \ldots, w_{n}(t)\right]^{T}$ is the vector of model innovations having zero mean and covariance matrix $\Sigma_{w}$. The autoregressive coefficients $a_{i j}(k), i, j=1, \ldots, n$ represent the 
linear interaction effect of $x_{j}(t-k)$ onto $x_{i}(t)$. In order to provide a frequency domain description of Granger-causality, Baccala and Sameshima [18] introduced the concept of Partial Directed Coherence (PDC) which has recently been generalized to the new PDC [82] as follows:

Let

$$
A(\lambda)=\sum_{k=1}^{p} A_{k} e^{-i 2 \pi \lambda k}
$$

be the Fourier transform of the coefficient matrices, where $\lambda$ is the normalized frequency in the interval $[-0.5,0.5]$ and $i=\sqrt{-1}$. Then the new PDC is defined as [82]

$$
\left|\pi_{i \leftarrow j}(\lambda)\right|=\frac{\frac{1}{\sigma_{i}}\left|\bar{A}_{i j}(\lambda)\right|}{\sqrt{\sum_{m=1}^{p} \frac{1}{\sigma_{m}^{2}} \bar{A}_{m j}(\lambda) * \bar{A}_{m j}^{H}(\lambda)}}
$$

where $\bar{A}(\lambda)=I-A(\lambda)$ and $\sigma_{i}^{2}$ refers to the variance of the innovation processes $w_{i}(t)$. $\left|\pi_{i \leftarrow j}(\lambda)\right|$ ranges between 0 (indicating independence) and 1 (indicating maximum coherence).

Phase Locking Value (PLV)

Phase synchronization presents a different approach in analyzing the possible nonlinear interdependencies of the EEG signal and focuses on the phases of the signals. The Phase Locking Value (PLV) is one of the most used robust phase coupling measures. It assumes that two dynamic systems may have their phases synchronized even if their amplitudes are zero correlated [83]. The phase synchronization (PS) is defined as the locking of the phases associated to each signal:

$$
\left|\phi_{x}(t)-\phi_{y}(t)\right|=\text { const }
$$

In order to estimate the instantaneous phase of our signal, we transform it using the Hilbert transform (HT), whereby the analytical signal $H(t)$ is computed as:

$$
H(t)=x(t)+i \tilde{x}(t)
$$

where $\tilde{x}(t)$ is the HT of $x(t)$, defined as:

$$
\tilde{x}(t)=\frac{1}{\pi} P V \int_{-\infty}^{\infty} \frac{x\left(t^{\prime}\right)}{t-t^{\prime}} d t^{\prime}
$$


where $P V$ denotes the Gauchy principal value. The analytical signal phase is defined as:

$$
\phi(t)=\arctan (\tilde{x}(t) / x(t))
$$

Therefore, for the two signals $x(t), y(t)$ of equal time length with instantaneous phases $\phi_{X}(t), \phi_{Y}(t)$, respectively, the PLV bivariate metric is defined below:

$$
P L V=\left|\frac{1}{N} \sum_{j=0}^{N-1} \exp \left(\mathrm{i}\left(\phi_{X}(j \Delta t)-\phi_{Y}(j \Delta t)\right)\right)\right|
$$

where $\Delta t$ is the sampling period and $N$ is the sample number of each signal. PLV takes values within the $[0,1]$ space, where 1 indicates perfect phase synchronization and 0 indicates lack of synchronization.

Nonlinear synchronization (state-space approach)

Finally, another group of synchronization measures are based on the assumption that neurons are highly nonlinear devices, which in some cases show chaotic behavior. Such measures belong to the generalized synchronization (GS) concept and are based on analyzing the interdependence between the amplitudes of the signals in a state-space reconstructed domain [84]. A physiological time series such as the EEG appears to have more than the single degree of freedom represented just by plotting the voltage as a function of time. To free up some of these unknown parameters, a standard technique is to map the scalar time series to a vector-valued one in a higher dimensional space $R^{m}$, thereby giving it an extension in space as well as time. Hence, one may measure how neighborhoods (recurrences) in state space located in one attractor map to each other. This idea turned out to be the most robust and reliable way of assessing the extent of GS [85]. First, we reconstruct delay vectors out of our time series:

$$
\mathbf{x}_{i}=\left[x_{i}, x_{i-\tau}, \quad, x_{i-(m-1) \tau}\right]^{T} \text { and } \mathbf{y}_{i}=\left[y_{i}, y_{i-\tau}, \quad, y_{i-(m-1) \tau}\right]^{T}
$$

where $i=1 \ldots N^{\prime}, N^{\prime}=N-(m-1) \tau$ and $m, \tau$ are the embedding dimension and time lag, respectively. Let $r_{i, j}$ and $s_{i, j}, j=1, \ldots, k$, denote the time indices of the $k$ nearest neighbors of $\mathbf{x}_{i}$ and $\mathbf{y}_{i}$, respectively. For each $\mathbf{x}_{i}$, the squared mean Euclidean distance to its $k$ neighbors is defined as:

$$
R_{i}^{(k)}(X)=\frac{1}{k} \sum_{j=1}^{k}\left(\mathbf{x}_{i}-\mathbf{x}_{r_{i, j}}\right)^{2}
$$

The $Y$-conditioned squared mean Euclidean distance $R_{i}^{(k)}(X \mid Y)$ is defined by replacing the nearest neighbors by the equal time partners of the closest neighbors of $\mathbf{y}_{i}$. If the set of reconstructed vectors (point cloud $\mathbf{x}_{i}$ ) has an average squared radius $R(X)=\left(1 / N^{\prime}\right) \sum_{n=1}^{N^{\prime}} R_{i}^{\left(N^{\prime}-1\right)}(X)$, then $R_{i}^{(k)}(X \mid Y) \approx R_{i}^{(k)}(X)<<R(X)$ if the systems are 
strongly correlated, while $R_{i}^{(k)}(X \mid Y) \approx R(X)>>R_{i}^{(k)}(X)$ if they are independent. Hence, an interdependence measure is defined as:

$$
S^{(k)}(X \mid Y)=\frac{1}{N^{\prime}} \sum_{i=1}^{N^{\prime}} \frac{R_{i}^{(k)}(X)}{R_{i}^{(k)}(X \mid Y)}
$$

Since $R_{n}^{(k)}(X \mid Y) \gg>R_{n}^{(k)}(X)$ by construction, it is clear that $S$ ranges between 0 (indicating independence) and 1 (indicating complete synchronization). Although, in general, $S^{(k)}(X \mid Y) \neq S^{(k)}(Y \mid X)$, this measure is not suitable to infer driver response relationships. Accordingly, we used the mean value $S(X ; Y)=\left(S^{(k)}(X \mid Y)+S^{(k)}(Y \mid X)\right) / 2$, as a Robust Interdependence Measure (RIM) between X and Y.

\section{BRAIN NETWORK VISUALIZATION AND APPLICATION DOMAINS}

Brain functional networks can be practically visualized by assigning to vertices the coordinates of the corresponding channels as described in section 2.1. Additionally, in order to show the network structure, one can use network visualization techniques such as the stress majorization technique of Gansner et al. [86] and the binary stress model of Koren et al. [87]. Apart from real world network visualization and graph analysis cases, there are some initial but indicative examples of possible translation of graph research outcomes in the clinical practice. More specifically, considering that the so called "disconnectivity syndromes" represent many pathological and neuropsychological diseases, based on their functional impairment symptoms, one can infer directly the usability and significance of network analysis and visualization tools based on graph theory. This section briefly discusses applications of graph visualization and analysis in cognitive and clinical domains, referring to published works of our group.

Rather recently, there is an interest in analyzing motor tasks using graphs in order to identify the involvement of different brain lobes. Such applications are central in the Brain-Computer Interface (BCI) field. Such visualizations allow us to select the most relevant sensors to take into account in the BCI classification tasks (i.e., focusing at those regions that act like hubs). Figure 3 depicts such networks using the PDC interdependence method applied at the mu frequency band [88]. Not only the interdependences but also the directionality of the coupling is illustrated using arrows [89].

Apart from the visualization itself, one is able to calculate and use the connectivity graphs to extract network properties and utilize them as biomarkers for certain pathologies such as alcoholism (Figure 4). In this case, synchronization was calculated using magnitude squared coherence and the standard 10/20. International montage along with an additional 41 sites as depicted in Figure 4 were used. Each subject was exposed to randomized pictures of objects chosen from the 1980 Snodgrass and Vanderwart picture set presented on a white background at the centre of a computer monitor and was requested to memorize and identify them at a later time. The results indicated that the proposed synchronization analysis in combination with the network analysis and visualization are able to picture with increased certainty the brain network topology during this working memory task. The alcoholic individual was found to have impaired synchronization of brain activity and loss of lateralization during the rehearsal process (Figure 4), most prominently in alpha band, as compared to a control subject [24]. What is interesting in this study is that there is no pre-specified threshold selection 
(a)

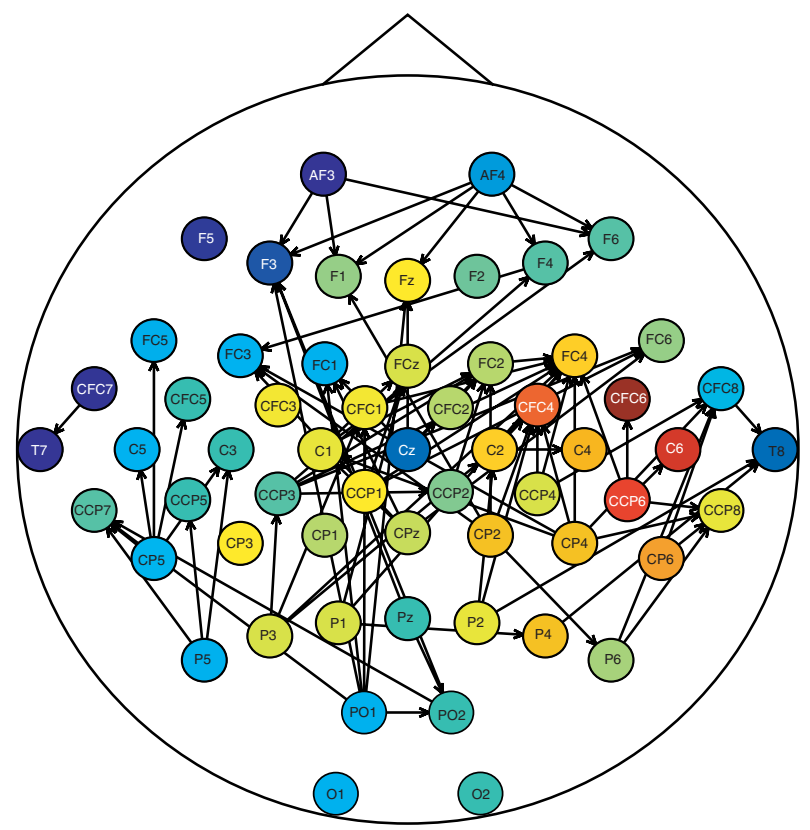

(b)

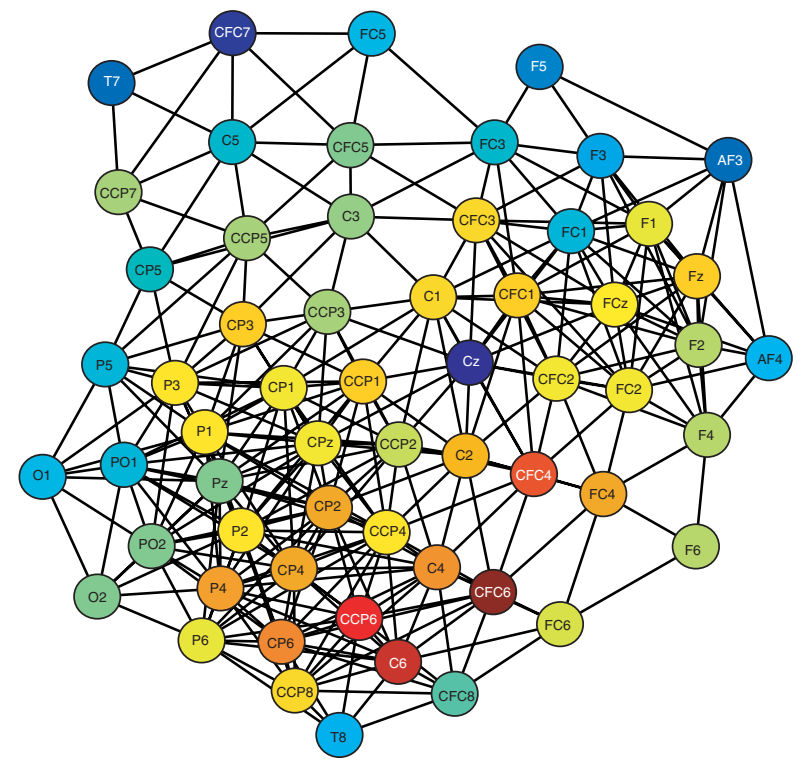

Figure 3. Brain network during foot movement imagination. (a) Network calculated with PDC at mu band, showing the edges that remain if the foot network is subtracted from the idle state network. The color of a vertex shows attributes of vertices, in this case depicting the Power Spectral Density (PSD) of channels at mu band. Red colors correspond to higher values than blue, whereas the color of a vertex shows the (average) PSD of channels. (b) Network calculated with multidimensional scaling at mu band [88]. 
(a)

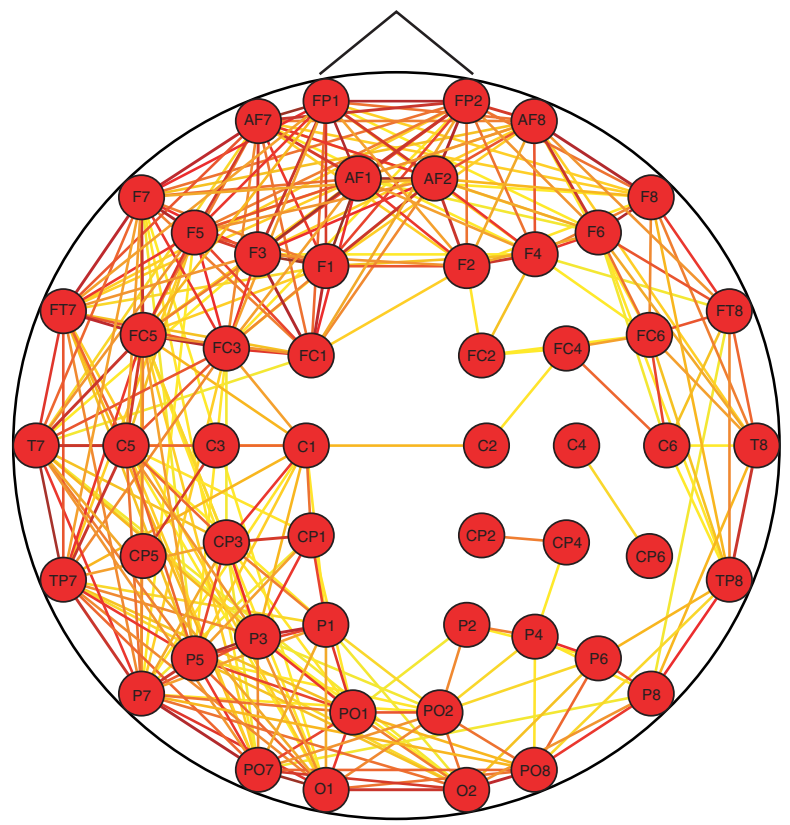

(b)

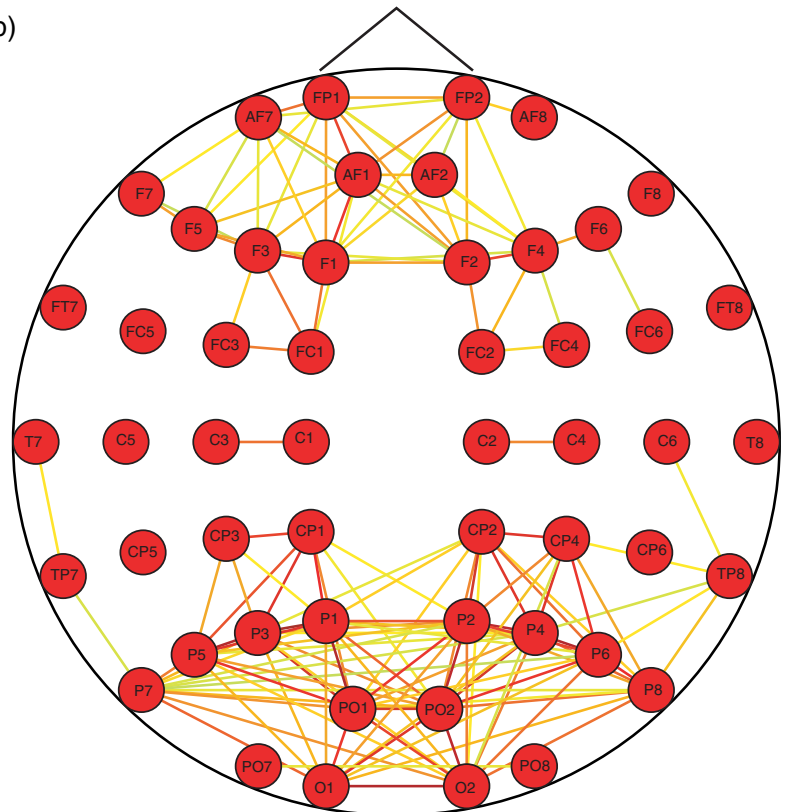

Figure 4. (a) Average network across trials of a control subject. (b) Average network across trials of an alcoholic subject. All networks were calculated at alpha band using magnitude squared coherence. Most significant connections are shown with red colors and wider edges. 


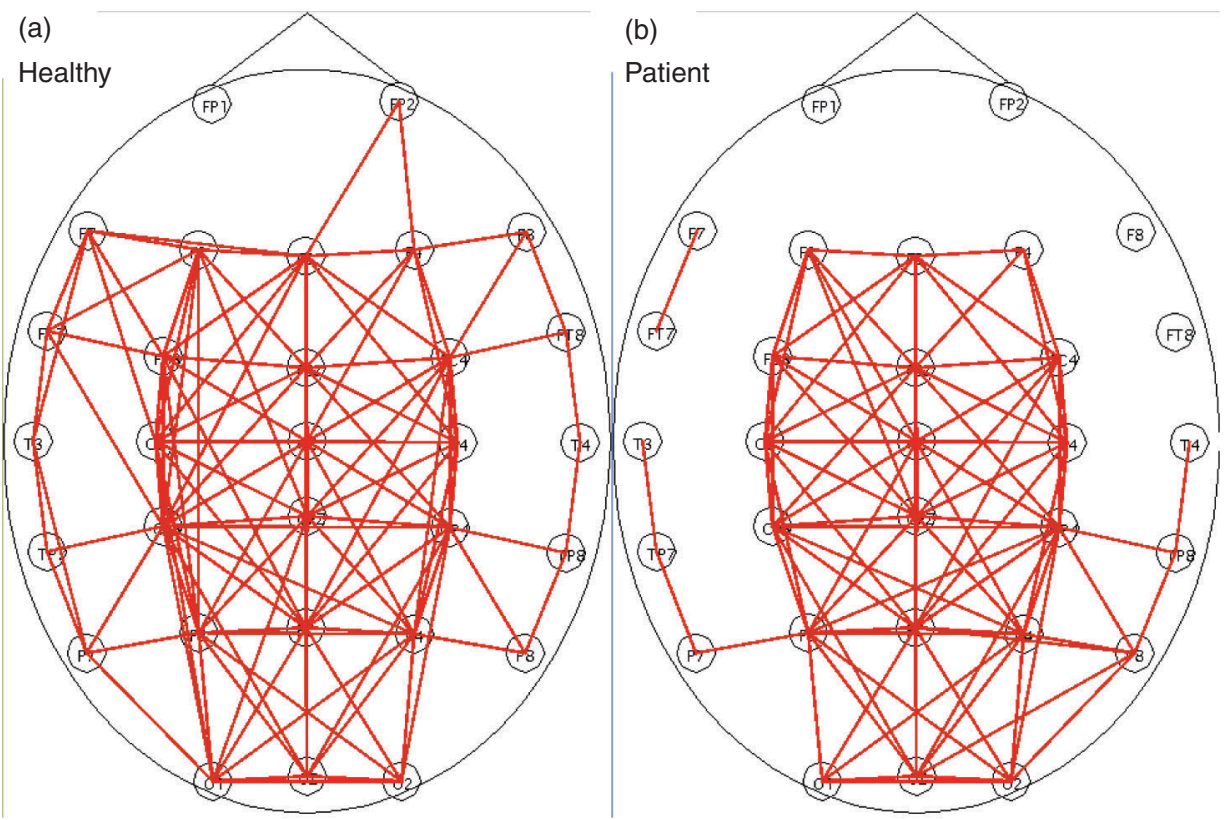

Figure 5. (a) A "healthy" brain network during a working memory task appears to have higher average degree $\mathrm{K}$ and clustering coefficient $\mathrm{C}$, and lower average shortest path length L values compared to the "schizophrenic" one (b). These disturbances are more prominent for the connections of the frontal lobes as well as the temporal lobes.

of edge drawing. Instead, threshold selection was based on testing 35 surrogate datasets to the null hypothesis from both the control and the alcoholic subjects for each channel pair. Only statistically significant edges reflecting all network connections are drawn differing in colour and width to reflect whether a strong (red shaded thick line) or weak (blue shaded thin line) interdependence is present (Figure 4). Focusing on the beta frequency band, impaired synchronization was also statistically tested using the clustering coefficient $\mathrm{C}$ parameter proving that the network topology of the alcoholic subject, as far as the $\mathrm{C}$ is concerned, is significantly less dense (t-test $\mathrm{p}$-value $<0.001)$ as compared to that of a control subject.

Using a similar experimental and computational analysis framework in studying schizophrenic activity under mental stress, the disconnection syndrome was evidenced (Figure 5). In this case, the EEG signals in both groups (20 controls and 20 stabilized patients with schizophrenia) were recorded from 30 cap electrodes, according to the $10 / 20$ international system. Interdependence was calculated using wavelet coherence that accounts for the non stationarity of the EEG signals as well. In this study the edge drawing threshold was selected according to $t$-test statistics applied on various properties of the resulting graph including the average degree $\mathrm{K}$, the clustering coefficient $\mathrm{C}$, and the average shortest path length $\mathrm{L}$ of our graph. The depicted topologies as modelled by the former network properties indicated higher levels of 
brain activity of schizophrenic populations than healthy ones. More direct connections among different functional lobes are needed when coping with mental tasks of increased difficulty [90].

Finally, as mentioned in the introduction one can also study neural interdependencies based on its independent components (ICs). Instead of directly measuring the synchronization using the actual EEG traces, ICs are first obtained and then identified based on their spatial and frequency properties using ICA decomposition in a concatenated trials scheme for each channel. Such approaches are most commonly used in analysing cognitive brain function. In the case of an auditory working memory paradigm (oddball experiment), induced responses are attributed to oscillatory bursts from local or distant neural assemblies with variable latency and frequency [91]. The functional coupling and role of independent components are investigated through the concept of PDC method. The EEG signals used in this work are selected from two representative subjects out of 9 healthy participants (age 37-74), who had no history of neurological or psychiatric disorder. This study indicates that functional connectivity during cognitive processes may be successfully assessed using connectivity measures applied on independent components, which reflect distinct spatial patterns of activity. The results suggest increased phase locked activity most prominently in the delta/ theta band, while alpha is also apparent in measures of non phase-locked activity [91]. Figure 6 depicts numbered ICs. Even though the control group involves a large variation in age, it has been demonstrated that processes related to working memory have been found to weaken with normal aging, but in general follow the same patterns of activation [92]. This is also verified in Figure 6 showing two similar networks of subjects of different ages. The strong interaction in the alpha band is indicated by directed lightblue lines, whereas the weak influence of the theta band from the alpha band is indicated with dashed lines. The independent components identified relate to the alpha, theta and delta bands on the basis of their (major) frequency activity. The delta components (related to cognitive processing) strongly relate to the alpha components.

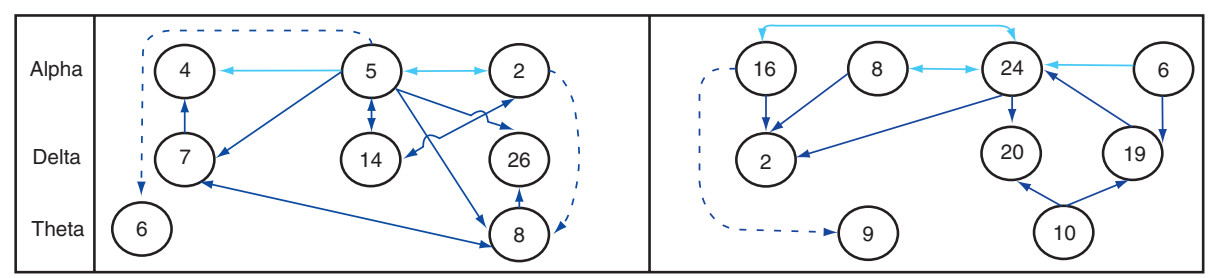

Figure 6. Networks of numbered ICs reflecting the synchronization maps of two representative subjects organized by means of the PDC of the band activity in Delta, Theta and Alpha. An auditory working memory paradigm (oddball experiment) induced responses are being attributed to oscillatory bursts from local or distant neural assemblies, with variable latency and frequency. Apart from the synchronization itself, directionality is also identified. The bidirectional coupling indicates no single influence between the "cause" and "effect" relationship [91]. 
In both cases, we can also observe two types of alpha components, an early one (associated with attention) at lower alpha band and a late one at the fast alpha range, with the early one driving the late alpha. The early alpha associated with attention drives a delta component related to cognition, which in turn drives the late alpha component that is also related to memory operations; this relationship in Figure 6 is observed as node influence from 5 to 7 to 4 in the left network and from 6 to 19 to 24 in the right network. Similar tendencies maybe easily identified using directed graphs based on directed coupling methods such as PDC.

Apart from the presented application domains, several studies have been conducted focusing on other pathologies like Alzheimer Disease [93], Epilepsy [94] and Parkinson's disease [95].

\section{CONCLUSIONS}

Our brain is a complex network in which information is continuously processed and transported between spatially distributed but functionally linked regions [1]. This ongoing integration of information enables us to evaluate the world around us and to respond quickly and flexibly to complex situations. A snapshot of a subject's dynamic brain functional network can be estimated from high resolution EEG measurements. Then this network can be characterized and analyzed using well formed concepts mainly based on graph theory and statistical physics. Our research has been facilitated by recent progresses in the development of efficient algorithms to calculate graph measures and to draw graphs. Graph visualization reveals the hidden structure of the networks and enhances human understanding, thus leading to new insights and new findings. To summarize, networks have emerged as a unifying theme for describing and investigating the functional connectivity. This approach has been very fruitful in theoretical neuroscience and remains to find its applications to clinical practice and other applications. For example, differences in graph indices between healthy and mentally impaired subjects could be used, together with other features, as "biomarkers" for medical diagnosis. In addition, to accompany this article, we also provide a freely accessible standalone brain visualization tool to benefit the healthcare engineering community. The toolkit implements a number of network indices and visualization techniques for graphs and can be accessed online (http://www.ics.forth.gr/bmi/tools.html). Hopefully, the healthcare community could greatly benefit from such visualization and graph analysis tools capable of revealing the hidden brain network structure.

\section{ACKNOWLEDGMENTS}

This work was supported in part by the EC Virtual Physiological Human Network of Excellence (VPH NoE).

\section{NOMENCLATURE}

$A_{k} \quad$ Coefficient matrix at lag $k$ of a multivariate autoregressive process of order $p, \operatorname{MVAR}(p)$

$A(\lambda) \quad$ Fourier transform of the coefficient matrices of an $\operatorname{MVAR}(p)$ process 
$a_{i j}(k) \quad$ Autoregressive coefficients. Elements of matrix $A_{k}$

$C \quad$ Clustering coefficient of a graph $G$

$C(v) \quad$ Clustering coefficient of a vertex $v \in V$

$C_{B}(v) \quad$ Shortest path betweenness centrality of a vertex $v \in V$

$C_{D}(v) \quad$ Degree centrality of a vertex $v \in V$

$C_{D}^{+}(v) \quad$ Out-Degree centrality of a vertex $v \in V$

$C_{D}^{-}(v) \quad$ In-Degree centrality of a vertex $v \in V$

$C_{E f}(v) \quad$ Shortest path efficiency of a vertex $v \in V$

$\operatorname{deg}(v) \quad$ The degree of a vertex $v \in V$

$\operatorname{deg}^{+}(v) \quad$ The out-degree of a vertex $v \in V$

$\operatorname{deg}^{-}(v) \quad$ The in-degree of a vertex $v \in V$

dest $(e) \quad$ The destination of an edge $e \in E$

$d_{G}(u, v) \quad$ The shortest path distance from vertex $u$ to vertex $v$

$E \quad$ Edges of a graph

$|E| \quad$ The cardinality of edges of a graph

Ef Efficiency of a graph $G$

$G \quad$ Graph

$H(t) \quad$ Analytical signal

$K \quad$ Average degree of a graph $G$

$L \quad$ Average shortest path length of a graph $G$

$M \quad$ Parameter used to denote the number of edges in random graph models, interdependence measures parameters

$m \quad$ Embedding dimension

$N \quad$ Number of samples of a time series

$N(v) \quad$ The set of neighbors of a vertex $v \in V$

$N^{+}(v) \quad$ The set of out-neighbors of a vertex $v \in V$

$N^{-}(v) \quad$ The set of in-neighbors of a vertex $v \in V$

$n \quad$ The cardinality of vertices of a graph $(n=|V|)$, or the number of simultaneously observed time series (viz. the number of channels).

$\operatorname{orig}(e) \quad$ The origin of an edge $e \in E$

PLV Phase locking value

$p \quad$ The order of a multivariate autoregressive process

$q \quad$ Probability used in random graph models

$r \quad$ Assortativity coefficient of a graph $G$

$S^{(k)}(X ; Y) \quad$ Robust interdependence measure between $X$ and $Y$

$S_{x y}(f) \quad$ Cross spectral density function

$\Delta t \quad$ The sampling period of a time series

$V \quad$ Vertices of a graph

$|V| \quad$ The cardinality (i.e. the number) of vertices of a graph

$\tilde{x}(t) \quad$ Hilbert transform of a signal $x(t)$

\section{Greek}

$\gamma_{x y}(f)$

Magnitude square coherence for a given frequency $f$

$\varepsilon(v)$

Eccentricity of a vertex $v$ in a connected graph $G$ 


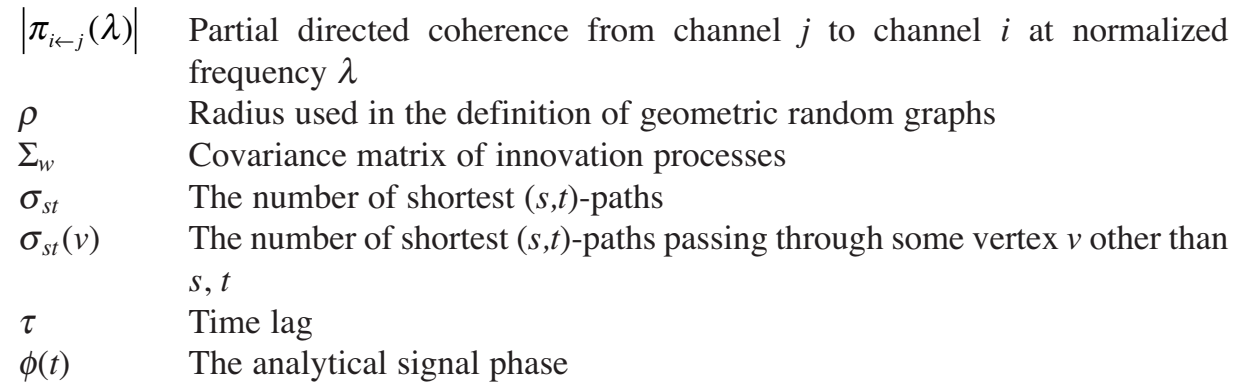

\section{REFERENCES}

[1] Sporns, O., Tononi, G. and Edelman, G.M., Connectivity and complexity: the relationship between neuroanatomy and brain dynamics, Neural Networks, 2000, 13, 909-922.

[2] Sporns, O., Chialvo, D.R., Kaiser, M. and Hilgetag, C.C., Organization, development and function of complex brain networks, Trends in Cognitive Sciences, 2004, 8(9), 418-425.

[3] Friston, K.J., Functional and effective connectivity in neuroimaging: A synthesis, Human Brain Mapping, 1994, 2(1-2), 56-78.

[4] Horwitz, B., The elusive concept of brain connectivity, NeuroImage, 2003, 19(2), 466-470.

[5] Fingelkurts, A.A., Fingelkurts, A.A. and Kähkönen, S., Functional connectivity in the brain-is it an elusive concept, Neuroscience \& Biobehavioral Reviews, 2005, 28(8), 827-836.

[6] Varela, F., Lachaux, J.P., Rodriguez, E. and Martinerie, J., The brainweb: Phase synchronization and large-scale integration, Nature Reviews Neuroscience, 2001, 2(4), 229-239.

[7] Bullmore, E. and Sporns, O., Complex brain networks: graph theoretical analysis of structural and functional systems, Nature Reviews Neuroscience, 2009, 10(3), 186-198.

[8] Honey, C.J., Kötter, R., Breakspear, M. and Sporns, O., Network structure of cerebral cortex shapes functional connectivity on multiple time scales, Proceedings of the National Academy of Sciences USA, 2007, 104(24), 10240-10245.

[9] Fox, M.D. and Raichle, M.E., Spontaneous fluctuations in brain activity observed with functional magnetic resonance imaging, Nature Reviews Neuroscience, 2007, 8(9), 700-711.

[10] Vincent, J.L., Patel, G.H., Fox, M.D., Snyder, A.Z., Baker, J.T., Essen, D.C.V., Zempel, J.M., Snyder, L.H., Corbetta, M. and Raichle, M.E., Intrinsic functional architecture in the anaesthetized monkey brain, Nature, 2007, 447(7140), 83-86.

[11] Bassett, D.S., Meyer-Lindenberg, A., Achard, S., Duke, T. and Bullmore, E., Adaptive reconfiguration of fractal small-world human brain functional networks, Proceedings of the National Academy of Sciences USA, 2006, 103(51), 19518-19523.

[12] Kaiser J. and Lutzenberger W., Induced gamma-band activity and human brain function, The Neuroscientist, 2003, 9(6), 475-484.

[13] Delorme A., Makeig S., Fabre-Thorpe M. and Sejnowski T., From Single-trial EEG to Brain Area Dynamics, Neurocomputing, 2002, 44-46, 1057-1064.

[14] Meinecke F.C., Ziehe A., Kurths J. and Müller K.R., Measuring Phase Synchronization of Superimposed Signals, Physical Review Letters, 2005, 94(8), 084102.

[15] Ioannides A.A., Dynamic Functional Connectivity, Current Opinion in Neurobiology, 2007, 17(2), 161-170.

[16] Sakkalis V., Tsiaras V., Michalopoulos K. and Zervakis M., Assessment of neural dynamic coupling and causal interactions between independent EEG components from cognitive tasks using linear and nonlinear methods, Proceedings of the 30th IEEE Annual International Conference on Engineering in Medicine and Biology Society, Vancouver, Canada, 2008, 3767-3770. 
[17] Sakkalis, V., Giurcăneanu, C.D., Xanthopoulos, P., Zervakis, M., Tsiaras, V., Yang, Y. and Micheloyannis, S., Assessment of linear and nonlinear synchronization measures for analyzing EEG in a mild epileptic paradigm, IEEE Transactions on Information Technology in Biomedicine, 2009, 13(4), 433-441.

[18] Baccalá, L.A. and Sameshima, K., Partial directed coherence: a new concept in neural structure determination, Biological Cybernetics, 2001, 84(6), 463-474.

[19] Strogatz, S. H., Exploring complex networks, Nature, 2001, 410, 268-276.

[20] Rubinov, M. and Sporns, O., Complex network measures of brain connectivity: Uses and interpretations, NeuroImage, In Press

[21] Uhlhaas, M.J. and Singer, W., Neural synchrony in brain review disorders: Relevance for cognitive dysfunctions and pathophysiology, Neuron, 2006, 52(1-2), 155-168.

[22] Seeley, W.W., Crawford, R.K., Zhou, J., Miller, B.L. and Greicius, M.D., Neurodegenerative diseases target large-scale human brain networks, Neuron, 2009, 62(1), 42-52.

[23] Fingelkurts, A.A., Fingelkurts, A.A. and Kähkönen, S., New perspectives in pharmacoelectroencephalography, Progress in Neuro-Psychopharmacology and Biological Psychiatry, 2005, 29(2), 193-199.

[24] Sakkalis, V., Tsiaras, V., Zervakis, M. and Tollis, I.G., Optimal brain network synchrony visualization: Application in an alcoholism paradigm, Proceedings of the IEEE 29th Annual International Conference on Engineering in Medicine and Biology Society, Lyon, France, 2007, 4285-4288.

[25] Niedermeyer, E., Epileptic seizure disorders, in: Niedermeyer, E., Lopes da Silva, F., eds., Electroencephalography: Basic Principles, Clinical Applications, and Related Fields, Lippincott Williams \& Wilkins, 2005, 505-620.

[26] Steriade, M., Neuronal Substrates of Sleep and Epilepsy, Cambridge University Press, Cambridge, UK, 1993.

[27] Stam, C.J., de Haan, W., Daffertshofer, A., Jones, B.F., Manshanden, I., van Cappellen van Walsum, A.M., Montez, T., Verbunt, J.P.A., de Munck, J.C., van Dijk, B.W., Berendse, H.W. and Scheltens, P., Graph theoretical analysis of magnetoencephalographic functional connectivity in Alzheimer's disease, Brain, 2009, 132(1), 213-224.

[28] Stam, C.J., Jones, B., Nolte, G., Breakspear, M. and Scheltens, P., Small world networks and functional connectivity in Alzheimer's disease, Cerebral Cortex, 2007, 17(1), 92-99.

[29] Jeong, J., EEG dynamics in patients with Alzheimer's disease, Clinical Neurophysiology, 2004, 115(7), 1490-1505.

[30] Just, M.A., Cherkassky, V.L., Keller, T.A., Kana, R.K. and Minshew, N.J., Functional and anatomical cortical underconnectivity in autism: Evidence from an fMRI study of an executive function task and corpus callosum morphometry, Cerebral Cortex, 2007, 17(4), 951-961.

[31] Murias, M., Webb, S.J., Greenson, J. and Dawson, G., Resting state cortical connectivity reflected in EEG coherence in individuals with autism, Biological Psychiatry, 2007, 62(3), 270-273.

[32] Rippon, G., Brock, J., Brown, C. and Boucher, J., Disordered connectivity in the autistic brain: Challenges for the 'new psychophysiology', International Journal of Psychophysiology, 2007, 63(2), 164-172.

[33] Hammond, C., Bergman, H. and Brown, P., Pathological synchronization in parkinson's disease: Networks, models and treatments, Trends in Neurosciences, 2007, 30(7), 357-364.

[34] Kubicki, M., McCarley, R., Westin, C.F., Park, H.J., Maier, S., Kikinis, R., Jolesz, F.A. and Shenton, M.E., A review of diffusion tensor imaging studies in schizophrenia, Journal of Psychiatric Research, 2007, 41(1-2), 15-30.

[35] Micheloyannis, S., Pachou, E., Stam, C., Breakspear, M., Bitsios, P., Vourkas, M., Erimaki, S. and Zervakis, M., Small-world networks and disturbed functional connectivity in schizophrenia, Schizophrenia Research, 2006, 87(1-3), 60-66.

[36] Liu, Y., Liang, M., Zhou, Y., He, Y., Hao, Y., Song, M., Yu, C., Liu, H., Liu, Z. and Jiang, T., Disrupted small-world networks in schizophrenia, Brain, 2008, 131(4), 945-961. 
[37] Rubinov, M., Knock, S.A., Stam, C.J., Micheloyannis, S., Harris, A.W.F., Williams, L.M. and Breakspear, M., Small-world properties of nonlinear brain activity in schizophrenia, Human Brain Mapping, 2009, 30(2), 403-416.

[38] van den Heuvel, M.P., Stam, C.J., Kahn, R.S. and Hulshoff Pol, H.E., Efficiency of functional brain networks and intellectual performance, The Journal of Neuroscience, 2009, 29(23), 7619-7624.

[39] Stam, C.J. and Reijneveld, J.C., Graph theoretical analysis of complex networks in the brain, Nonlinear Biomedical Physics, 2007, 1(3).

[40] Pachou, E., Vourkas, M., Simos, P., Smit, D., Stam, C.J., Tsirka, V. and Micheloyannis, S., Working memory in schizophrenia: An EEG study using power spectrum and coherence analysis to estimate cortical activation and network behavior, Human Brain Mapping, 2008, 21(2), 128-137.

[41] Massimini, M., Ferrarelli, F., Huber, R., Esser, S.K., Singh, H. and Tononi, G., Breakdown of cortical effective connectivity during sleep, Science, 2005, 309(5744), 2228-2232.

[42] He, Y., Chen, Z.J. and Evans, A.C., Small-world anatomical networks in the human brain revealed by cortical thickness from MRI, Cerebral Cortex, 2007, 17(20), 2407-2419.

[43] Watts, D.J. and Strogatz, S.H., Collective dynamics of 'small-world' networks, Nature, 1998, 393(6684), 440-442.

[44] Hage, P. and Harary, F., Eccentricity and centrality in networks, Social Networks, 1995, 17(1), 57-63.

[45] Brandes, U. and Erlebach, T., eds, Network Analysis Methodological Foundations, Lecture Notes in Computer Science, Springer, 2005, 3418.

[46] He, Y., Dagher, A., Chen, Z., Charil, A., Zijdenbos, A., Worsley, K. and Evans, A., Impaired smallworld efficiency in structural cortical networks in multiple sclerosis associated with white matter lesion load, Brain, 2009, 132(12), 3366-3379.

[47] Achard, S. and Bullmore, E., Efficiency and cost of economical brain functional networks, PLoS Computational Biology, 2007, 3(2), e17.

[48] Newman, M.E.J., Assortative mixing in networks, Physical Review Letters, 2002, 89(20), 208701.

[49] Newman, M.E.J., Mixing patterns in networks, Physical Review E, 2003, 67(2), 026126.

[50] Newman, M.E.J. and Park, J., Why social networks are different from other types of networks, Physical Review E, 2003, 68(3), 036122.

[51] Barabási, A.L. and Oltvai, Z.N., Network biology: understanding the cell's functional organization, Nature Reviews Genetics, 2004, 5(2), 101-113.

[52] Durek, P. and Walther, D., The integrated analysis of metabolic and protein interaction networks reveals novel molecular organizing principles, BMC System Biology, 2008, 2(100).

[53] Park, C.H., Kim, S.Y., Kim, Y.H. and Kim, K., Comparison of the small-world topology between anatomical and functional connectivity in the human brain, Physica A, 2008, 387(23), 5958-5962.

[54] Maslov, S. and Sneppen, K., Specificity and stability in topology of protein networks, Science, 2002, 296(5569), 910-913.

[55] Xulvi-Brunet, R. and Sokolov, I.M., Reshufling scale-free networks: From random to assortative, Physical Review E, 2004, 70(6), 066102.

[56] Yu, H., Kim, P.M., Sprecher, E., Trifonov, V. and Gerstein, M., The importance of bottlenecks in protein networks: Correlation with gene essentiality and expression dynamics, PLoS Computational Biology, 2007, 3(4), e59.

[57] Jordán, F., Benedek, Z. and Podani, J., Quantifying positional importance in food webs: A comparison of centrality indices, Ecological Modelling, 2007, 205(1-2), 270-275.

[58] Hagmann, P., Cammoun, L., Gigandet, X., Meuli, R., Honey, C.J., Wedeen, V.J. and Sporns, O., Mapping the structural core of human cerebral cortex, PLoS Biology, 2008, 6(7), e159.

[59] Albert, R. and Barabási, A.L., Statistical mechanics of complex networks, Reviews of Modern Physics, 2002, 74(1), 47-97.

[60] Manke, T., Demetrius, L. and Vingron, M., An entropic characterization of protein interaction networks and cellular robustness, $J$ R Soc Interface, 2006, 3(11), 843-850. 
[61] Latora, V. and Marchiori, M., Efficient behavior of small-world networks, Physical Review Letters, 2001, 87(19), 198701.

[62] Latora, V. and Marchiori, M., Economic small-world behavior in weighted networks, The European Physical Journal B, 2003, 32(2), 249-263.

[63] Freeman, L.C., A set of measures of centrality based upon betweenness, Sociometry, 1977, 40(1), 35-41.

[64] Brandes, U., A faster algorithm for betweenness centrality, Journal of Mathematical Sociology, 2001, 25(2), 163-177.

[65] Solomonoff, R. and Rapoport, A., Connectivity of random nets, Bulletin of Mathematical Biophysics, $1951,13,107$.

[66] Erdös, P. and Rényi, A., On random graphs I, Publicationes Mathematicae (Debrecen), 1959, 6, 290-297.

[67] Erdös, P. and Rényi, A., On the evolution of random graphs, Publ. Math. Inst. Hung. Acad. Sci., 1960, 5 A $17-61$.

[68] Bollobás, B., Random Graphs, Cambridge University Press, Cambridge, UK, 2001.

[69] van den Heuvel, M., Stam, C., Boersma, M. and Pol, H.H., Small-world and scale-free organization of voxel-based resting-state functional connectivity in the human brain, NeuroImage, 2008, 43(3), 528-539.

[70] Barabási A.-L., Albert R. and Jeong H., Mean-field theory for scale-free random networks, Physica A, 1999, 272, 173-187.

[71] Barabási A.-L. and Albert R., Emergence of scaling in random networks, Science, 1999, 286, 509-512.

[72] Broder, A., Kumar, R., Maghoul, F., Raghavan, P., Rajagopalan, S., Stata, R., Tomkins, A. and Wiener, J., Graph structure in the web, Computer Networks, 2000, 33(1-6), 309-320.

[73] Jeong, H., Tombor, B., Albert, R., Oltvai, Z.N. and Barabási, A.L., The large scale organization of metabolic networks, Letters to Nature, 2000, 407(6804), 651-654.

[74] Jeong, H., Mason, S.P., Barabási, A.L. and Oltvai, Z.N., Lethality and centrality in protein networks, Nature, 2001, 411(6833), 41-42.

[75] Eguíluz, V.M., Chialvo, D.R., Cecchi, G.A., Baliki, M. and Apkarian, A.V., Scale-free brain functional networks, Physical Review Letters, 2005, 94(1), 018102.

[76] Achard, S., Salvador, R., Whitcher, B., Suckling, J. and Bullmore, E., A resilient, low-frequency, small-world human brain functional network with highly connected association cortical hubs, The Journal of Neuroscience, 2006, 26(1), 63-72.

[77] Iturria-Medina, Y., Sotero, R.C., Canales-Rodríguez, E.J., Alemán-Gómez, Y. and Melie-García, L., Studying the human brain anatomical network via diffusion-weighted MRI and graph theory, NeuroImage, 40(3), 2008, 1064-1076.

[78] Penrose, M., Random Geometric Graphs, Oxford University Press, 2003.

[79] Higham, D.J., Rašajski, M. and Pržulj, N., Fitting a geometric graph to a protein-protein interaction network, Bioinformatics, 2008, 24(8), 1093-1099.

[80] Oikonomou, T., InBrAiN: An Interactive Tool for Brain Analysis and Visualization. Master's thesis, Computer Science Department, University of Crete, 2007, http://elocus.lib.uoc.gr

[81] Granger, C.W.J., Investigating causal relations by econometric models and cross-spectral methods, Econometrica, 1969, 37(3), 424-438.

[82] Baccalá, L.A. and Sameshima, K., Generalized partial directed coherence, Proceedings of the 15th IEEE International Conference on Digital Signal Processing, Cardiff, UK, 2007, 163-166.

[83] Rosenblum, M., Pikovsky, A., Kurths, J., Schäfer, C. and Tass, P.A., Phase synchronization: from theory to data analysis, in: Neuro-Informatics and Neural Modelling, Handbook of Biological Physics, North Holland, 2001, 4.

[84] Sauer, T., Yorke, J.A. and Casdagli, M., Embedology, Journal of Statistical Physics, 1991, 65(3-4), 579-616. 
[85] Arnhold, J., Grassberger, P., Lehnertz, K. and Elger, C.E., A robust method for detecting interdependences: application to intracranially recorded EEG, Physica D, 1999, 134(4), 419-430.

[86] Gansner, E.R., Koren, Y. and North, S., Graph drawing by stress majorization, Proceedings of the 12th International Symposium on Graph Drawing, LNCS Springer-Verlag, New York, USA, 2004, 3383, $239-250$

[87] Koren, Y. and Civril, A.: The binary stress model for graph drawing. In: Tollis, Proceedings of the 16th International Symposium on Graph Drawing, LNCS Springer-Verlag, Heraklion, Greece, 2008, 5417, 193-205.

[88] Tsiaras, V., Andreou, D. and Tollis, I.G., Brainnetvis: Analysis and visualization of brain functional networks, Proceedings of the 31th Annual International Conference on Enginnering in Medicine and Biology Society, Minneapolis, USA, 2009, 2911-2914.

[89] Neumann, J., Fox, P.T., Turner, R. and Lohmann, G., Learning partially directed functional networks from meta-analysis imaging data, NeuroImage, 2010, 49(2), 1372-1384.

[90] Sakkalis, V., Oikonomou, T., Pachou, E., Tollis, I., Micheloyannis, S. and Zervakis, M., Timesignificant wavelet coherence for the evaluation of schizophrenic brain activity using a graph theory approach, Proceedings of the 28th Annual International Conference on Enginnering in Medicine and Biology Society, New York, USA, 2006, 4265-4268.

[91] Michalopoulos, K., Sakkalis, V., Iordanidou, V. and Zervakis, M., Activity detection and causal interaction analysis among independent EEG components from memory related tasks, Proceedings of the 31th Annual International Conference on Enginnering in Medicine and Biology Society, Minneapolis, USA, 2009, 2070-2073.

[92] Karrasch, M., Laine, M., Rapinoja, P. and Krause, C.M., Effects of normal aging on event-related desynchronization/synchronizatio during a memory task, Neuroscience Letters, 2004, 366(1), 18-23.

[93] He, Y., Chen, Z. and Evans, A.C., Structural insights into aberrant topological patterns of large-scale cortical networks in Alzheimer's disease, The Journal of Neuroscience, 2008, 28(18), 8148-8159.

[94] Schindler, K.A., Bialonski, S., Horstmann, M.T., Elger, C.E. and Lehnertz, K., Evolving functional network properties and synchronizability during human epileptic seizures, Chaos, 2008, 18(3), 033119 .

[95] Stoffers, D., Bosboom, J.L., Wolters, E.C., Stam, C.J. and Berendse, H.W., Dopaminergic modulation of cortico-cortical functional connectivity in Parkinson's disease: An MEG study, 2008, Experimental Neurology, 213(1), 191-195. 


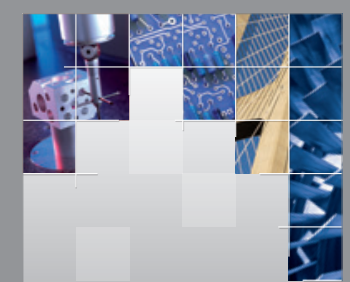

\section{Enfincering}
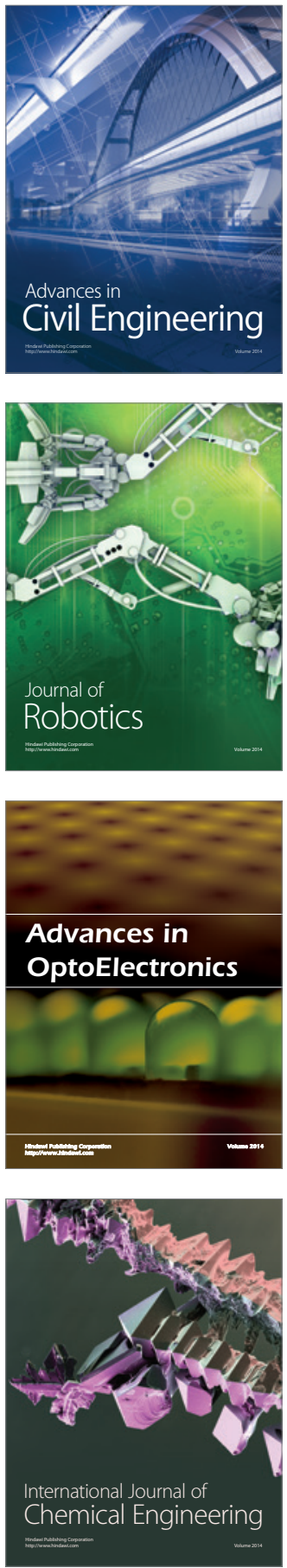

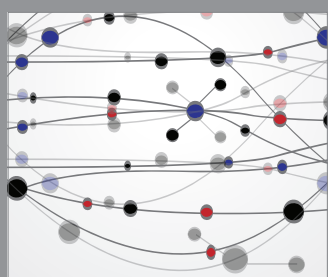

The Scientific World Journal

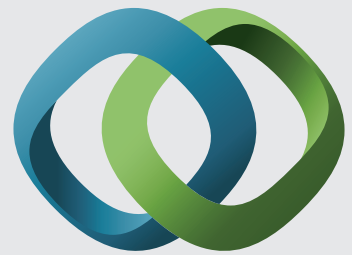

\section{Hindawi}

Submit your manuscripts at

http://www.hindawi.com
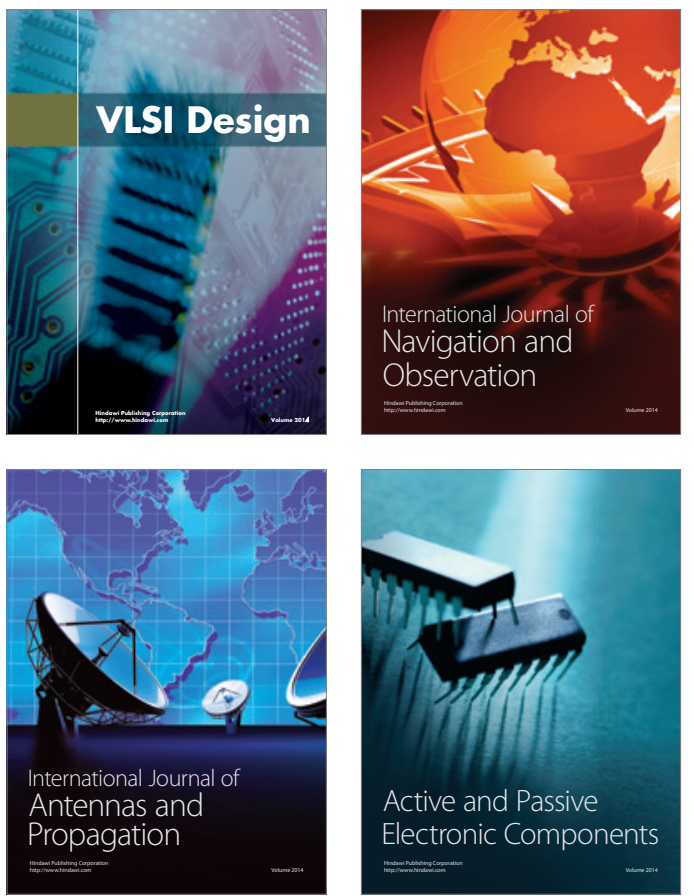
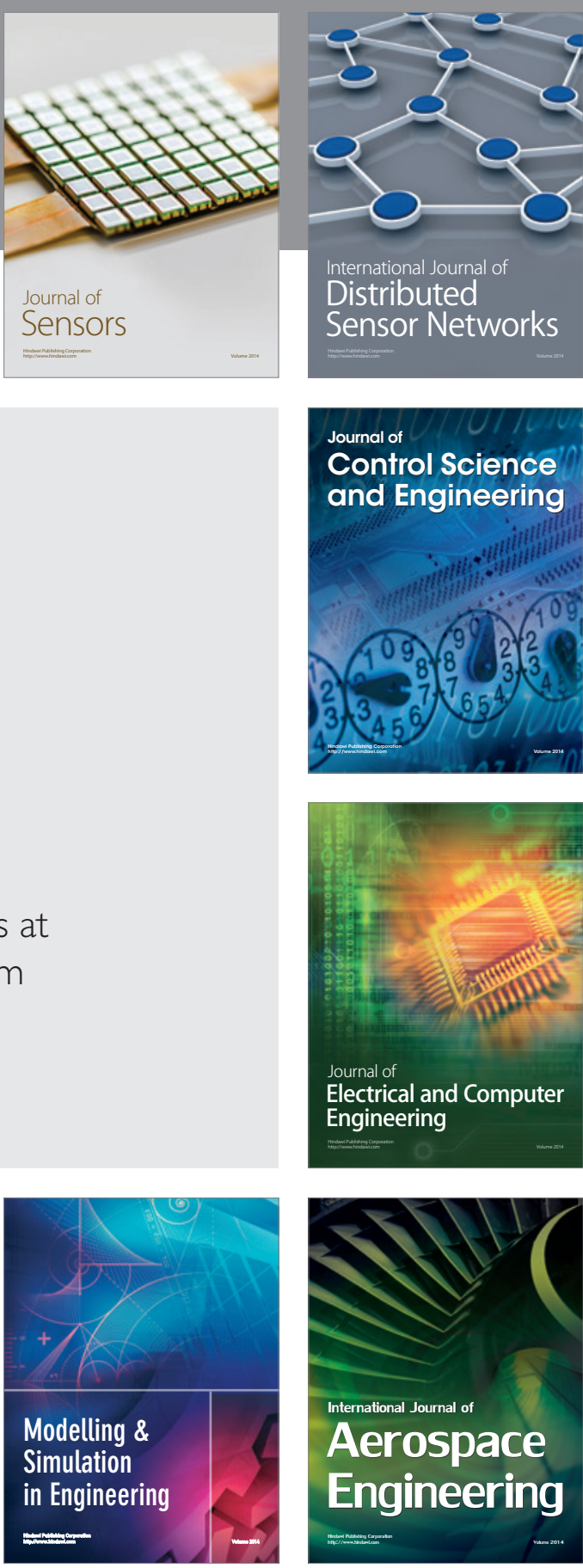

International Journal of

Distributed

Sensor Networks

Journal of

Control Science

and Engineering
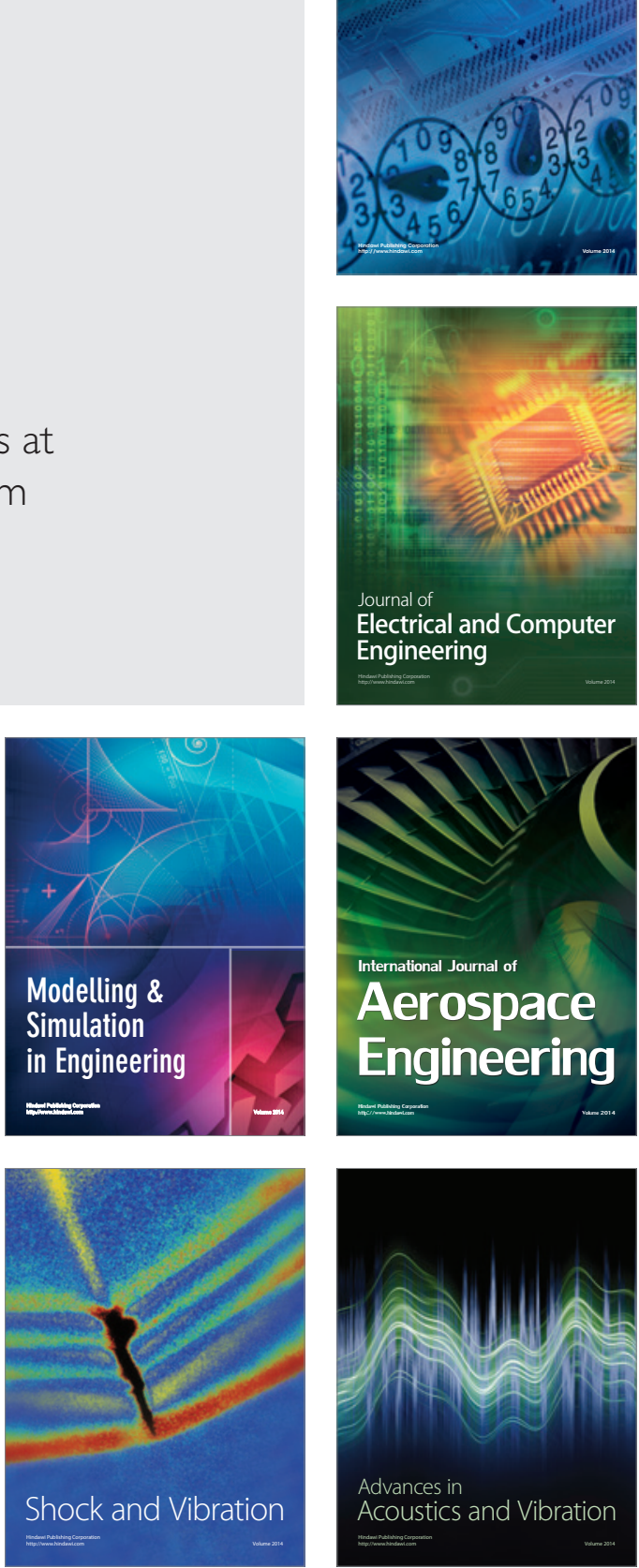